\title{
Group A streptococcal RofA-type global regulators exhibit a strain-specific genomic presence and regulation pattern
}

\author{
Bernd Kreikemeyer, ${ }^{1}$ Susanne Beckert, ${ }^{2}$ Andrea Braun-Kiewnick ${ }^{1}$ \\ and Andreas Podbielski ${ }^{1}$
}

1 Department of Medical Microbiology, Virology and Hygiene, University Hospital Rostock, Schillingallee 70, D-18055 Rostock, Germany

2 Department of Medical Microbiology and Hygiene, University Hospital Ulm, Robert-Koch-Str. 8, D-89091 Ulm, Germany

\author{
Author for correspondence: Bernd Kreikemeyer. Tel: +49 381494 5900. Fax: +493814945902. \\ e-mail: Bernd.Kreikemeyer@med.uni-rostock.de
}

RofA-like protein (RALP) type regulators have been shown to exist in different forms in group A streptococci (GAS) and to regulate the expression of important bacterial adhesins. This study shows that the vast majority of strains from different GAS M serotypes carried a rofA virulence regulator gene in their genome and that this gene could be detected in combination with other RALP genes and RALP-dependent adhesin genes in a strain-specific manner. The gene encoding the Nra regulator was predominantly found in opacity factor (OF)negative serotypes. When analysing a rofA mutant in a serotype $M 2$ strain, the strain specificity was also found in the positive and negative regulatory functions of RALP genes as well as in the type and number of virulence genes and functions controlled by the RALP genes. Of 17 virulence-associated genes tested, only one, the putative streptolysin $S$ gene, was observed to be derepressed in RALP mutants of three different GAS serotype strains. This strain-specific variability of RALP regulon sizes is associated with different patterns of host cell attachment and internalization. In addition, RofA2 was shown to control expression of the ribosomal protein gene rpsL. As a consequence, it was demonstrated for the first time in streptococci that aminoglycoside resistance mediated by rpsL expression is apparently controlled by a virulence gene regulator.

Keywords: Streptococcus pyogenes, response regulator, virulence gene regulation, adherence and internalization, bacteria-host cell interaction

\section{INTRODUCTION}

Group A streptococci (GAS; Streptococcus pyogenes) cause a large variety of diseases in their human host, including suppurative infections of the pharynx and skin, and severe invasive manifestations. The organism can also exist in an asymptomatic carrier state. The ability to enter the host and colonize several different anatomical sites can be explained by coordinated and differentiated regulation of virulence factors in response to the local environment.

The recently completed genome sequence of serotype M1 revealed the presence of 13 potential two-component

Abbreviations: GAS, group A streptococci; MSCRAMM, microbial surface components recognizing adhesive matrix molecules; OF, opacity factor; RALP, RofA-like protein. signal transduction systems that may contribute to this differential regulation. Only six of these putative regulators can be assigned to specific functions based on sequence homology (Ferretti et al., 2001). Of these, only the two sensor kinase/response regulator pairs CsrR/S (Levin \& Wessels, 1998; Bernish \& van de Rijn, 1999; Heath et al., 1999; Federle et al., 1999) and FasBCAX (Kreikemeyer et al., 2001) have been studied in any detail. Besides the obvious sensor-regulator pairs, GAS encode a notably high number of global regulators without known cognate sensors.

The RopB protein is required for transcription of the gene encoding cysteine protease SpeB (Lyon et al., 1998) and several other genes expressed primarily during stationary phase of growth (Chaussee $e$ t al., 2001). Mga is a global positive transcriptional regulator that controls genes encoding the $\mathrm{M}$ - and $\mathrm{M}$-related proteins, the fibronectin-binding serum opacity factor (OF), the 
cysteine protease SpeB, the C5a peptidase, the complement inhibiting factor Sic and the collagen-like proteins SclA and SclB (Perez-Casal et al., 1991; Chen et al., 1993; Podbielski et al., 1995; McLandsborough \& Cleary, 1995; Kreikemeyer et al., 1999; Lukomski et al., 2000a,b, Rasmussen et al., 2000; Lukomski et al., 2001; Rasmussen \& Björck, 2001). The Mga regulator exists in two versions with about $70 \%$ sequence homology. The Mga type is associated with specific serotypes.

The RofA-like protein (RALP) regulator family comprises four different versions with a mean of $52 \%$ deduced amino acid sequence similarity and $29 \%$ identity (Granok et al., 2000). Unlike the mga gene, several RALP regulators can be encoded by a single GAS strain (Podbielski et al., 1999; Granok et al., 2000; Ferretti et al., 2001). However, the two best studied RALP-type regulators, RofA in a serotype M6 strain and $\mathrm{Nra}$ in a serotype M49 strain, occurred alone. RofA was first described as a positive transcriptional regulator of the gene encoding the fibronectin-binding protein $(p r t F)$ in GAS serotype M6 strains (Fogg et al., 1994; Fogg \& Caparon, 1997). RofA is autoregulated and responds to increased oxygen levels (VanHeyningen et al., 1993). Since PrtF/SfbI is a major adhesin of GAS and also promotes internalization of the bacteria by epithelial cells (Hanski \& Caparon, 1992; Talay et al., 1991, 1992, 1994; Molinari et al., 1997; Molinari \& Chhatwal, 1999), RofA function is also important for bacteria-host cell interactions (Beckert et al., 2001). As well as its positive regulatory effects, RofA was recently shown to suppress transcription of speA, sagA and $m g a$ (Beckert et al., 2001). Therefore, RofA could be the repressor of $m g a$, whose existence was recently suggested by McIver et al. (1999). Two RofA-binding boxes have been experimentally identified in the rofA-prtF intergenic region. Putative RofA-binding boxes were identified upstream of at least one other gene in the serotype M1 genome sequence (Granok et al., 2000).

In contrast to RofA, the Nra pattern was shown to be that of a negative regulator in GAS. The nra gene is located adjacent to a collagen-binding adhesin (cpa)gene in serotype M49 strains (Podbielski et al., 1999). The $\mathrm{Nra}$ regulon includes a gene encoding a second fibronectin-binding protein, PrtF2 (Jaffe et al., 1996). Investigation of a small number of GAS serotypes indicated several combinations of $n r a / r o f A, p r t F / p r t F 2$ and cpa genes (Podbielski et al., 1999). The type of genes regulated by RALP family members suggested an involvement in GAS-eukaryotic cell interactions exceeding the known functions of protein F. In fact, Nra regulation contributes to a balanced expression of virulence factors and avoidance of host cell damage, thereby promoting GAS persistence in eukaryotic cells (Molinari et al., 2001).

It is possible that RALP family members could have more specific regulatory functions in particular serotypes than other GAS regulators. First, either one, two or several RALP genes can be found in combinations with various MSCRAMM (microbial surface components recognizing adhesive matrix molecules; Patti et al., 1994) genes like prtF, prtF2 and $c p a$. Second, also the number and type of genes contained in a RALP regulon appear to be serotype-dependent.

To elucidate the presence of RALP-encoding genes and MSCRAMM-encoding genes in diverse GAS serotypes, 62 isolates were tested in the present study for the presence of rofA, $n r a, p r t F, p r t F 2$ and $c p a$ genes. Subsequently, a serotype M2 strain was chosen for functional analysis of its rof $A$ gene because of the clinical importance of M2 strains e. g. in Germany (Brandt et al., 2001) and because of the unusual structure of the core RALP regulon. To establish a nomenclature for rof and nra genes with respect to different $M$ serotypes, the rofA-like gene in M2 was designated as rofA2 from here on and rofA-like genes from other serotypes were designated accordingly. With three different serotypes now examined for the genes and functions under control of a single RALP-encoding gene, the differences between a common and the serotype-specific pattern of RALP function have begun to emerge.

\section{METHODS}

Bacterial strains and culture conditions. GAS strains were grown in THY at $37^{\circ} \mathrm{C}$ in a $5 \% \mathrm{CO}_{2}$ atmosphere. The different $\mathrm{M}$ serotype GAS strains used in this study have been described by Podbielski et al. $(1991,1993)$ and Kaufhold et al. (1992). Production of serum OF was tested as described by Johnson \& Kaplan (1988). All mutant strains were maintained on medium supplemented with $60 \mathrm{mg}$ spectinomycin $\mathrm{l}^{-1}$. The RofA6 and Nra49 mutant strains have been described elsewhere (Beckert et al., 2001; Molinari et al., 2001). The EmmLuc recombinant strain was constructed in this study according to methods published by Podbielski et al. (1999).

Nucleic acid techniques. Construction of the pSF152-rofA6 plasmid used for insertional inactivation of the rofA2 allele was described by Beckert et al. (2001). The Qiagen Mini Prep kit was used to prepare plasmid DNA from Escherichia coli. Preparation of competent $S$. pyogenes cells and transformation of GAS M2 followed the protocol of Caparon \& Scott (1991). The presence of single plasmids in the M2 chromosome and the correct location of the inserted plasmid was confirmed by Southern blot techniques, PCR and Northern blot hybridizations. Chromosomal DNA from GAS was prepared according to Martin et al. (1990).

Genomic DNA of 62 serotype strains was digested with EcoRI or HindIII and, after agarose gel electrophoresis, blotted onto Pall Biodyne B membrane. For hybridization Dig-dUTP labelled PCR products were generated with primers described by Natanson et al. (1995), Jaffe et al. (1996) and Podbielski et al. (1999). In addition, the same primers were used for direct PCR assays using genomic DNA as template to amplify rofA, $n r a, p r t F, p r t F 2$ and cpa genes. This analysis only included well characterized genes and not other so far uncharacterized RALP members identified by Granok et al. (2000).

Amplification of DNA by PCR was performed according to standard protocols (Ausubel et al., 1990). For the generation of DNA probes for DNA-DNA and DNA-RNA hybrid- 
ization, $0 \cdot 5 \mu \mathrm{DIG}-\mathrm{dUTP}$ was added to the reaction mixture. Agarose gel electrophoresis, DNA transfer to membranes, Southern blots and detection of hybridizing bands was carried out as described by Podbielski et al. (1995). The RNA preparation followed the protocol of Shaw \& Clewell (1985). Denaturing agarose gel electrophoresis and Northern Blot hybridization with Dig-dUTP-labelled probes were as previously described (Podbielski et al., 1995). DIG-dUTP-labelled gene probes of virulence factor and housekeeping genes for DNA-RNA hybridizations were generated by PCR, using various primers that were designed on the basis of published sequences.

Inverse PCR and sequencing. M2 chromosomal DNA (5 $\mu \mathrm{g})$ was digested with HindII. Digested DNA was purified according to standard methods (Ausubel et al., 1990) and was used undiluted, and at dilutions of $1: 10$ and $1: 100$ for ligation. Ligation mixtures $(10 \mu$ l total volume) were incubated overnight at $16{ }^{\circ} \mathrm{C}$. Five microlitres of each ligation mixture were used as template for subsequent PCR amplification using primer RofAInvFor (5'-AGAAACTTATCTTTACCAACTCTA-3') and RofAInvRev (5'-TTCGATTGATGATTCCAAG-3'). The Expand High Fidelity system (Roche) was used and PCR conditions were as follows: 35 cycles each consisting of 1 min denaturation at $94^{\circ} \mathrm{C}, 1 \mathrm{~min}$ annealing at $50{ }^{\circ} \mathrm{C}$ and $3 \mathrm{~min} 30 \mathrm{~s}$ extension at $72^{\circ} \mathrm{C}$. A $1.5 \mathrm{~kb}$ DNA band was amplified from all ligation mixtures. Sequencing of the purified regular and inverse PCR products was performed by MWG Biotech by utilizing the PCR primers as sequencing primers. Homology searches were performed with the global alignment algorithm described by Person et al. (1997).

Functional assays. Determination of haemolytic activity contained in supernatants of wild-type and RofA2 mutant bacteria and measurement of binding capacity towards extracellular matrix and plasma proteins was performed as described previously (Kreikemeyer et al., 2001). Culture and maintenance of the human laryngeal epithelial cell line HEp2 (ATCC CCL23,) and adherence and invasion assays using wild-type and mutant bacteria were essentially as described by Molinari et al. (2001). For all functional assays described above three individual tests were performed on three different days. Data shown are the means of such assays with standard deviations $( \pm S D)$ indicated. Determination of MICs employed the E test according to the manufacturer's instructions (AB Biodisk).

Statistical analyses. The Mann-Whitney $U$ test was used to determine if the mean values of the datasets from the functional assays are significantly different. The significance level $(\alpha)$ at which the test was performed is indicated in the main text of the appropriate section.

\section{RESULTS}

\section{Presence of RALP and MSCRAMM genes in 61 GAS serotypes}

To examine the occurrence of different RALP- and MSCRAMM-encoding genes in a collection of diverse GAS serotypes we applied PCR and hybridization techniques to chromosomal DNA isolated from 62 strains (representing 61 different $M$ serotypes). All MSCRAMM-encoding genes known to be members of RALP regulons were included $(p r t F, p r t F 2$ and $c p a)$. However, for RALP-encoding genes the study focussed only on the functionally characterized members (rofA6 and nra49) and excluded the uncharacterized ralP3 and ralP5 genes (Granok et al., 2000). The data are summarized in Table 1.

Fifty-two $(83 \%)$ of the strains tested harboured a rof $A$ gene, whereas only $17(27 \%)$ carried the nra gene. Of these 17 strains, seven $(41 \%)$ contained both rofA and nra genes. Only three OF-positive strains carried a copy of $n \mathrm{ra}$. In contrast, fourteen OF-negative strains were nra-positive, suggesting $n r a$ to be more common among OF-negative strains. The $p r t F$ gene was present in $70 \%$ of the strains tested. A comparable frequency as well as the association of $p r t F$ with OF-positive serotypes was also reported by Natanson et al. (1995) and Goodfellow et al. (2000). A total of $53 \%$ of the strains carried a prtF2 gene and $32 \%$ harboured a cpa gene. The majority of strains (52 out of 62) carried one or two MSCRAMMencoding genes. No MSCRAMM gene was detected in serotype M2, M3, M52 and M57 strains.

All possible combinations of RALP- and MSCRAMMencoding genes were found. Additionally, no association was detected between RALP/MSCRAMM patterns and specific diseases commonly associated with particular GAS serotypes.

\section{Construction of a rofA mutant in the GAS serotype M2}

Based on the epidemiological importance of this serotype (Brandt et al., 2001), the presence of only a rofA gene, and the unusual RALP- and MSCRAMM-encoding gene pattern, an M2 serotype strain was chosen for further characterization to investigate the strain specificity of RALP-mediated regulation. Using regular and inverse PCR strategies we were able to amplify the rofA 2 gene and to determine its sequence. The sequence showed a $98.3 \%$ identity with the rofA6 gene sequence (accession number U01312; Fogg et al., 1994). The corresponding nra49 gene sequence (accession number U49397; Podbielski et al., 1999) exhibited only $62.6 \%$ sequence homology. The rofA2 gene was inactivated by insertion mutagenesis (Beckert et al., 2001) and the mutation was confirmed by PCR and Southern hybridization (data not shown). No differences in the growth rate and final optical cell density between wild-type and mutant was detected (data not shown).

\section{Characterization of extracellular matrix- and plasma- protein binding activity of the M2 wild-type and RofA mutant}

One of the main features of RALP family members is their involvement in transcriptional regulation of fibronectin binding (M6 and M49) and collagen-binding adhesins (M49). Expression of these MSCRAMMs directly influences important biological functions, such as bacteria-host cell interactions.

Therefore, we investigated the extracellular and plasma protein-binding capacity of the wild-type M2 strain and the RofA2 mutant at the early exponential, late exponential and stationary phases of growth (Table 2). Only low levels of fibrinogen binding were observed for 
Table 1. Distribution of RALP- (rofA and nra) and MSCRAMM- (prtF, prtF2 and cpa) encoding genes among 62 strains of 61 different $M$ serotypes

-, Absence; +, presence of the gene, as judged by PCR and Southern hybridization experiments. For details of the assay refer to Methods. OF column: +, positive; - , negative. Numbers in bold depict total number of strains positive for the tested gene, and in parenthesis the percentage of the total strains tested is given.

\begin{tabular}{|c|c|c|c|c|c|c|}
\hline M serotype & OF & $\operatorname{rof} A$ & nra & $p r t F$ & $p r t F 2$ & cpa \\
\hline 1 & - & + & - & - & - & + \\
\hline 2 & + & + & - & - & - & - \\
\hline 3 & - & - & + & - & - & - \\
\hline 4 & + & + & + & + & + & - \\
\hline 5 & - & - & + & - & + & - \\
\hline 6 & - & + & - & + & - & - \\
\hline 6 & - & + & - & + & - & - \\
\hline 8 & + & + & - & - & - & + \\
\hline 9 & + & + & - & + & + & + \\
\hline 11 & + & + & - & + & + & + \\
\hline 12 & - & + & - & + & + & - \\
\hline 13 & + & + & - & + & + & - \\
\hline 14 & - & + & - & + & + & + \\
\hline 15 & + & + & - & + & - & - \\
\hline 17 & - & + & - & + & - & - \\
\hline 18 & - & - & + & - & + & - \\
\hline 19 & - & + & - & + & - & - \\
\hline 22 & + & + & - & + & + & + \\
\hline 23 & - & + & - & + & - & - \\
\hline 24 & - & + & - & + & - & - \\
\hline 25 & + & + & - & + & - & + \\
\hline 26 & - & + & - & + & - & - \\
\hline 27 & - & + & - & + & - & - \\
\hline 28 & + & + & - & + & + & - \\
\hline 29 & - & + & - & + & - & + \\
\hline 30 & - & + & - & + & - & + \\
\hline 31 & - & + & - & + & - & - \\
\hline 32 & - & + & + & + & + & + \\
\hline 33 & - & - & + & - & + & - \\
\hline 34 & - & - & + & - & + & - \\
\hline 36 & - & + & + & - & + & + \\
\hline 37 & - & + & - & + & - & - \\
\hline 38 & - & + & - & + & - & - \\
\hline 39 & - & - & + & + & + & - \\
\hline 40 & + & + & - & + & - & - \\
\hline 41 & - & + & - & + & - & + \\
\hline 42 & - & - & + & - & + & + \\
\hline 43 & - & - & + & - & + & + \\
\hline 44 & + & + & - & + & + & - \\
\hline 46 & - & + & - & + & - & - \\
\hline 47 & - & + & - & + & + & + \\
\hline 48 & + & + & - & + & - & - \\
\hline 49 & + & - & + & - & + & + \\
\hline 50 & - & + & - & + & + & - \\
\hline 51 & - & + & - & + & + & - \\
\hline 52 & - & + & + & - & - & - \\
\hline 53 & - & + & + & - & + & - \\
\hline 54 & - & + & - & + & - & - \\
\hline 55 & - & + & - & + & + & - \\
\hline
\end{tabular}


Table 1 (cont.)

\begin{tabular}{|lcccccc|}
\hline M serotype & OF & rof $A$ & nra & prtF & prtF2 & cpa \\
\hline 56 & - & + & + & - & + & + \\
57 & - & + & + & - & - & - \\
58 & + & + & - & + & - & - \\
59 & + & - & + & + & + & - \\
60 & + & + & - & + & + & - \\
61 & + & + & - & + & + & - \\
62 & + & + & - & + & + & - \\
65 & - & + & - & + & - & - \\
66 & + & + & - & + & + & - \\
67 & - & + & - & - & + & + \\
69 & - & + & - & - & + & + \\
71 & - & + & - & - & + & + \\
None-typeable & - & + & - & + & - & - \\
$(64 / 14)$ & & & & & & \\
Total: 62 & $+\mathbf{2 0}$ & $\mathbf{5 2}$ & $\mathbf{1 7}$ & $\mathbf{4 3}$ & $\mathbf{3 3}$ & $\mathbf{2 0}$ \\
& $-\mathbf{4 2}$ & $(\mathbf{8 3} \%)$ & $(\mathbf{2 7} \%)$ & $(\mathbf{7 0} \%)$ & $(\mathbf{5 3} \%)$ & $(\mathbf{3 2} \%)$ \\
\hline
\end{tabular}

Table 2. Extracellular and plasma protein binding by a serotype M2 GAS strain and its isogenic RofA2 mutant

The assay was performed as described by Kreikemeyer et al. (1995). Results from three independent assays are shown. The Mann-Whitney $U$ test was used to calculate statistical significance. The percentage binding was calculated as bound c.p.m. $\times 100 /$ added c.p.m.

\begin{tabular}{|llrrr|}
\hline \multirow{2}{*}{ Protein and strain type } & \multicolumn{3}{c|}{ Percentage binding* $^{*}$} \\
\cline { 3 - 5 } & & \multicolumn{1}{c|}{$\mathbf{3} \mathbf{h}$} & $\mathbf{8} \mathbf{h}$ & Overnight \\
\hline \multirow{2}{*}{ Fibronectin } & Wild-type & $22 \cdot 3 \pm 1 \cdot 2$ & $29 \cdot 1 \pm 2 \cdot 1$ & $19 \cdot 1 \pm 0 \cdot 9$ \\
& Mutant & $23 \cdot 3 \pm 0 \cdot 4$ & $14 \cdot 1 \pm 0 \cdot 8$ & $14 \cdot 9 \pm 0 \cdot 6$ \\
Fibrinogen & Wild-type & $9 \cdot 5 \pm 0 \cdot 9$ & $9 \cdot 5 \pm 0 \cdot 9$ & $7 \cdot 3 \pm 0 \cdot 4$ \\
& Mutant & $11 \cdot 3 \pm 0 \cdot 1$ & $7 \cdot 5 \pm 0 \cdot 4$ & $6 \cdot 5 \pm 0 \cdot 3$ \\
Collagen I & Wild-type & $32 \cdot 1 \pm 3 \cdot 4$ & $42 \cdot 8 \pm 0 \cdot 6$ & $41 \cdot 1 \pm 0 \cdot 2$ \\
& Mutant & $41 \pm 0 \cdot 6$ & $38 \cdot 8 \pm 1 \cdot 3$ & $39 \cdot 9 \pm 1 \cdot 8$ \\
\hline
\end{tabular}

$*$ Mean \pm SD.

the wild-type and mutant at any phase of growth. In contrast, high level binding of collagen type I was detected. The rofA 2 mutation increased collagen binding by $10 \%$ in early exponential phase. However, the difference was not statistically significant $(\alpha, 5 \%)$ and the binding activity of both the wild-type and mutant were the same during the later stages of growth. Binding of fibronectin was not significantly different in the wildtype and mutant bacteria from early exponential phase, but the RofA2 mutant showed a significant $(\alpha, 5 \%) 50 \%$ decrease in fibronectin binding during late exponential phase. In cells harvested from late stationary phase cultures a significant difference in binding capacity was still detectable. These results suggested that RofA in M2 acted as a positive regulator of fibronectin-binding activity in agreement with earlier observations with the RofA6 mutant (Fogg et al., 1994; Beckert et al., 2001).
The PCR and Southern blotting analyses described in Table 1 indicated that the well characterized $p r t F$ and prtF2 genes encoding fibronectin-binding proteins were absent from strain M2. Therefore we performed PCR analyses and Southern hybridization experiments using primers and probes directed against $f b p 54$ and sof/sfbII. These two MSCRAMM-encoding genes have been shown to encode proteins capable of fibronectin binding (Rakonjac et al., 1995; Kreikemeyer et al., 1995, 1999; Courtney et al., 1994, 1999; Katerov et al., 2000), and might therefore be responsible for the fibronectinbinding activity observed in this serotype. With both experimental approaches the presence of $f b p 54$ and sof/sfbII genes was verified (data not shown). It has never been investigated if either of these genes is controlled by a RALP-type regulator. Strains lacking PrtF and PrtF2 have been described (Natanson et al., 
Table 3. Summary of transcriptional analysis of genes in defined RofA/Nra mutants

The data are accumulated from published results (Fogg et al., 1994; Podbielski et al., 1999; Beckert et al., 2001; Molinari et al., 2001) and this study. 0 , transcription not affected; - , decreased transcription; +, increased transcription; NT, not tested; NP, not present in serotype.

\begin{tabular}{|c|c|c|c|c|c|c|c|c|c|c|c|c|c|c|c|c|c|c|c|}
\hline \multirow{2}{*}{$\begin{array}{l}\text { RALP } \\
\text { mutant }\end{array}$} & \multicolumn{2}{|c|}{$\begin{array}{c}\text { Housekeeping } \\
\text { genes }\end{array}$} & \multicolumn{7}{|c|}{ Surface expressed virulence factors } & \multicolumn{5}{|c|}{ Secreted virulence factors } & \multicolumn{5}{|c|}{ Regulatory genes } \\
\hline & $\operatorname{rec} A$ & $r p s L$ & cpa & $f b p 54$ & $\mathrm{emm}$ & prtF2 & prtF & sof & has B & spe $A$ & speB & $\operatorname{sag} A$ & slo & ska & fas $X$ & $m g a$ & nra & $c s r$ & $\operatorname{rof} A$ \\
\hline Nra M49 & NT & NT & + & + & 0 & + & NP & + & 0 & + & + & + & 0 & 0 & NT & + & + & NT & NP \\
\hline RofA M6 & NT & NT & NP & NT & + & NP & - & $\mathrm{NP}$ & NT & + & 0 & + & 0 & NT & NT & + & NP & NT & - \\
\hline RofA M2 & 0 & - & $\mathrm{NP}$ & 0 & - & NP & NP & 0 & - & 0 & 0 & + & 0 & + & 0 & 0 & NP & 0 & - \\
\hline
\end{tabular}

(a) $\operatorname{rof}$

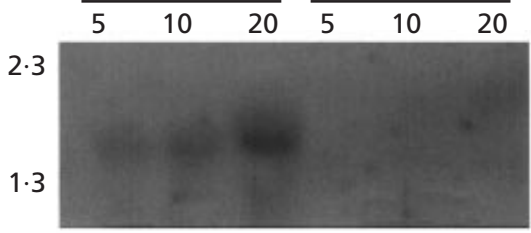

(b) emm

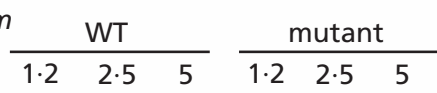

$1 \cdot 3$

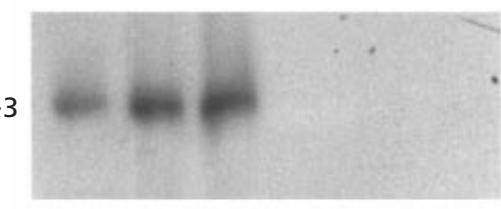

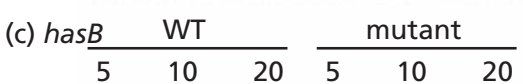

$4 \cdot 4$

$2 \cdot 3$

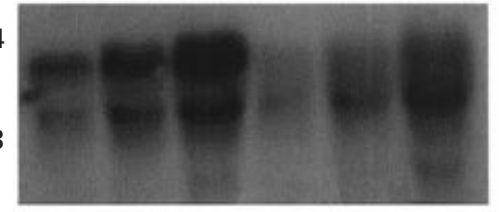

(d) $\operatorname{rec} A \quad$ WT

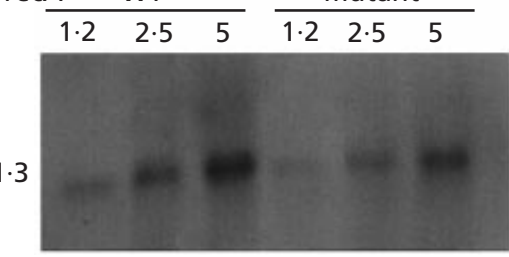

(e) $\operatorname{fas} X$

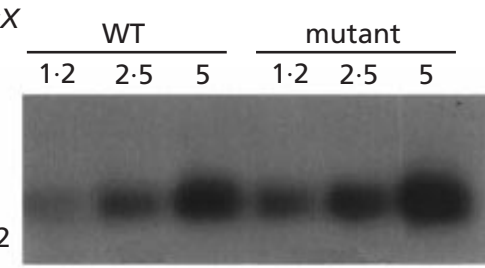

(i) $s p e A \frac{\text { WT }}{10} \quad \begin{array}{ll}\text { mutant } \\ \end{array}$

$1 \cdot 3$

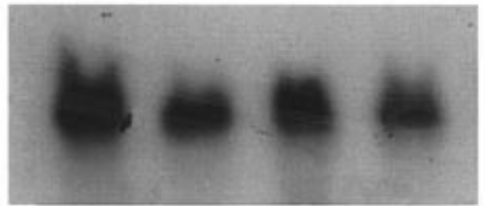

(f) $\operatorname{sag} A / p e$

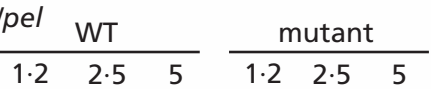

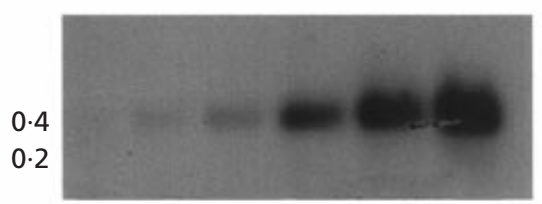

(j) mga

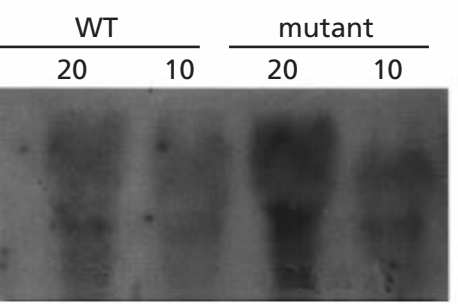

(g) $s k a$

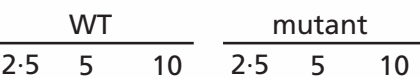

$2 \cdot 3$

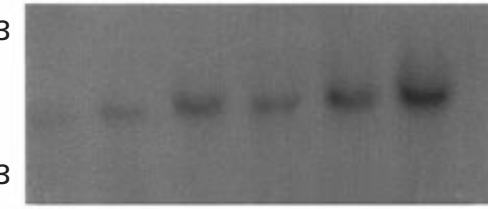

(k) $f b p 54$

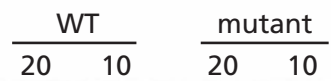

$2 \cdot 3$

$1 \cdot 3$

(h) $r p s L$
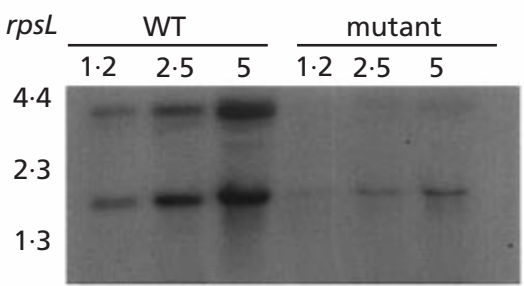

(I) sof2

$$
\begin{array}{llll}
\frac{\text { WT }}{20} \quad 10 & & \text { mutant } \\
\hline 20 \quad 10
\end{array}
$$

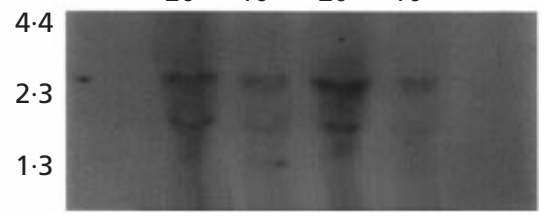

Fig. 1. Northern blot transcriptional analysis of the serotype $M 2$ GAS strain (wild-type; WT) and the isogenic RofA2 mutant strain (mutant). For preparation, quantification, electrophoresis, hybridization and detection of RNA refer to Kreikemeyer et al. (2001). DNA probes used are indicated above each part. Results shown are representative of those from at least three independent RNA isolations. The amounts $(\mu \mathrm{g})$ of RNA loaded in each lane are indicated above each lane. Molecular masses $(\mathrm{kb})$ of the RNA bands are indicated on the left hand side of each panel.

1995), but were not studied with respect to RALP regulation.

These results raised the question about the genomic environment of the rof $A$ gene in the M2 serotype, since in other serotypes either $p r t F$ or $c p a$ have been found adjacent to the rof $A$ gene. To test this, the region between the $5^{\prime}$ end of the lepA gene (putative signal peptidase I; Podbielski et al., 1999) and the $5^{\prime}$ end of the rofA2 gene was amplified as a fragment of $600 \mathrm{bp}$ suggesting the absence of $p r t F$ or $c p a$ genes. A similar experiment using a lep $A$ and nra specific primer pair on 
Table 4. Antibiotic resistance measured by the $E$ test (AB Biodisk)

The RofA2 mutant and EmmLuc strains were constructed in this study; all other strains are described by Podbielski et al. (1999), Beckert et al. (2001) and Molinari et al. (2001). The data shown are representative of three independent assays. NT, Not tested. Values represent $\mathrm{mg}^{-1}$

\begin{tabular}{|lcc|}
\hline GAS strain & Gentamicin & Streptomycin \\
\hline M2 WT & 16 & 32 \\
RofA2 mutant & 96 & 64 \\
M6 WT & 32 & $\mathrm{NT}$ \\
RofA6 mutant & 16 & $\mathrm{NT}$ \\
M49 WT & 12 & $\mathrm{NT}$ \\
Nra49 mutant & 12 & $\mathrm{NT}$ \\
EmmLuc & 12 & $\mathrm{NT}$ \\
\hline
\end{tabular}

M49 DNA generated a $3 \cdot 3 \mathrm{~kb}$ fragment, consistent with the presence of $c p a$ in this serotype (data not shown).

\section{Effects of rofA inactivation on expression of selected genes and biological functions}

The RALP family members in serotype M6 and M49 control a variety of virulence genes at the transcriptional level (Podbielski et al., 1999; Beckert et al., 2001) (see also Table 3). Therefore, our next objective was to determine how the rofA mutation in serotype M2 would influence gene expression (Fig. 1, Table 3). No truncated rofA2 message was detected in the mutant strain, indicating an at least 8-fold lower transcription in the mutant (Fig. 1a). This suggested a positive autoregulatory effect of RofA2, a fact that was also noted in the RofA6 mutant (Beckert et al., 2001). The genes encoding $M$ protein (emm; Fig. 1b), capsule synthesis enzymes (hasB; Fig. 1c), and ribosomal small subunit protein S12 (rpsL; Fig. 1h) were also found to be under positive control of RofA in serotype M2. RofA6 and RofA2 both acted as negative regulators of $m g a$ transcription (Beckert et al., 2001; Fig. 1j), but unlike in M6, emm transcription in M2 appears not to be directly coupled to transcription of $m g a$, since contrasting effects on emm were observed in the two serotypes.

In GAS, $r p s L$ has been used as a constitutively expressed control gene (Federle et al., 1999). Mutations in rpsL caused resistance to, and in some cases dependence on, streptomycin (Toivonen et al., 1999; Björkman et al., 1999). Thus, we investigated how downregulation of rpsL affected aminoglycoside resistance in the wild-type and mutant strain. Table 4 shows that downregulation of $r p s L$ caused increased MIC values for gentamicin and streptomycin in the RofA2 mutant. This effect can be explained by the decreasing number of target sites for aminoglycosides in the RofA2 mutant and is independent of the spectinomycin resistance determinant used for generating the mutant, since the MICs for gentamicin in the unrelated spectinomycin-resistant mutants were unaltered (Table 4).
The genes encoding the two secreted virulence factors, streptokinase $(s k a)$ and streptolysin $\mathrm{S}(\mathrm{pel} / \mathrm{sagA} / \mathrm{sls})$, were under negative transcriptional control of RofA in strain M2 (Fig. 1f, g). Enhanced expression of the gene encoding streptolysin $S$ was verified in a functional assay. The supernatant of the RofA2 mutant contained a significantly $(\alpha, 5 \%)$ higher haemolytic activity $(148 \pm 6 \cdot 8 \%)$ compared to the M2 wild-type bacteria $(100 \pm 4 \cdot 5 \%)$, with the haemolytic activity contained in supernatants from wild-type arbitrarily set to $100 \%$. Transcription of speA in strain M2 was not affected by the rofA2 mutation (Fig. 1i), in contrast to serotypes M6 and M49, where RofA6 and Nra49, respectively, caused speA to be transcribed at higher levels, suggesting negative control. Judged from their unchanged expression levels, recA, fas $X$ (Fig. 1d, e), $c s r R / S$ and the gene encoding SpeB (data not shown) are not part of the rof 22 regulon. Of note, the fibronectin-binding proteins encoded by $f b p 54$ and sof 2 did not appear to be regulated by RofA2 (Fig. 1k, l), suggesting an unidentified fibronectin-binding mechanism that is RALP-dependent. A comparison of the housekeeping and virulence gene transcription levels in RofA or Nra mutants from M2, M6 and M49 is summarized in Table 3.

\section{Influence of the rofA mutation on eukaryotic cell adherence and internalization}

Adherence to and internalization into cultured epithelial cells in vitro is a well established feature of $S$. pyogenes (Molinari \& Chhatwal, 1999). The processes are mediated by a fibronectin bridge which links bacterial cells to integrins on the epithelial cell surface and induces macro-pinocytosis (Molinari et al., 1997; Okada et al., 1998; Ozeri et al., 1998). Focal complex-like structures and recruitment of kinases from at least two signalling pathways facilitate the invasion process (Ozeri et al., 2001). Results presented in Table 2 suggest that RofA 2 is a positive regulator of fibronectin binding. If this is true, the RofA2 mutant would be less adhesive and invasive compared to the wild-type bacteria. Therefore, we investigated the influence of the RofA2 regulatory effects on cell adherence and internalization by performing antibiotic protection assays as described previously (Molinari et al., 2001). Unexpectedly, the RofA2 mutant was significantly $(\alpha, 1 \%)$ more adherent to the cultured HEp- 2 cells than the wild-type strain $(187 \pm 55 \%$ for the mutant; wild-type adherence was arbitrarily set to $100 \%)$. In contrast, the internalization rate of the mutant into HEp-2 cells compared to the wild-type was not significantly changed $(1.41 \pm 0.84 \%$ for the mutant; $0.9 \pm 0.5 \%$ for the wild-type).

\section{DISCUSSION}

The study described in this paper had two major objectives. First, we intended to examine the occurrence of different RALP- and MSCRAMM-encoding genes in a diverse collection of different GAS serotypes to determine the most frequent patterns of gene com- 
binations. Second, we intended to find common patterns of regulation by RALP-encoding genes in order to predict the association of patterns of RALP/ MSCRAMM genes and biological properties of specific GAS serotype strains.

The present investigation revealed that the most frequent representative of RALP genes is rofA, which was present in $83 \%$ of the GAS strains tested. An nra-like gene was found in $27 \%$ of the strains. In two-thirds of these strains nra and rofA occurred together. Each of the two RALP-encoding genes, either alone or in combination with other RALP-encoding genes, occurred in combination with one, two or even three RALPdependent MSCRAMM-encoding genes. Based on the analysis of 61 different GAS serotypes there is no obvious pattern of RALP- and RALP-dependent MSCRAMMencoding genes. This observation also applies to any potential link between gene patterns and the association of GAS serotypes with non-suppurative diseases like acute rheumatic fever or acute glomerulonephritis. Based upon the detection of anti-PrtF antibodies in sera from GAS-infected patients, Goodfellow et al. (2000) were also unable to demonstrate any association between antibody levels and the type of infection. We detected the $p r t F$ gene in $70 \%$ of the isolates tested. This number is in agreement with earlier reports by Kreikemeyer et al. (1995), Natanson et al. (1995) and Goodfellow et al. (2000), who reported $83 \%, 66 \%$ and $64 \%$ of isolates, respectively, to be prtF positive. In accordance with Goodfellow et al. (2000) we demonstrated a positive association between the occurrence of a $p r t F$ gene and the expression of serum OF. The only OF-positive strains without a detectable $\operatorname{prtF}$ gene were the serotype M2, M8 and M49 strains, of which the more thoroughly characterized M2 strain was the only one without any detectable MSCRAMM-encoding gene. Taken together, the presence or absence of specific RALP- or MSCRAMM-encoding genes seems to have little influence on the capacity of a GAS strain to cause purulent or non-suppurative diseases. The high number of detectable combinations among the different types of genes in the various serotypes indicates an ongoing and efficient lateral transfer of these genes.

To approach the second major objective of this study, an analysis of the rofA function in the serotype M2 strain using insertion mutagenesis appeared to be most promising. Downstream effects potentially caused by the insertion appeared very unlikely due to the monocistronic transcription mode of the rofA2 allele and presence of transcription terminators on the inserted vector. However, collateral damage caused by the rofA2 mutation could not be completely excluded, since complementation experiments were not attempted in this study. In trans complementation of response regulator mutants often leads to potentially ambiguous results due to gene dosage effects. In fact, functional analysis of a nra49 mutant (Molinari et al., 2001) that was complemented in trans did not result in restoration of the wild-type phenotype but in different gene expression patterns (B. Kreikemeyer \& A. Podbielski, unpublished results). Genetic systems for in cis chromosomal reconstitution are currently not available for GAS.

So far, the presence of a RALP-dependent MSCRAMM gene adjacent to the cognate RALP-encoding gene was regarded as a key feature of the genomic environment of this regulator family. Using different experimental approaches, we showed that the $p r t F$ and $c p a$ genes did not lie upstream of the rofA locus in the M2 serotype strain. Thus, such a strain was best suited when investigating the minimum congruency between RALP functions in a variety of GAS strains. Regardless of the absent $p r t F$ or cpa gene, the M2 wild-type strain exhibited strong collagen type I- and fibronectin-binding, indicating the presence of other surface factors with matrix-protein-binding capacity in the M2 strain. This also demonstrated a functional redundancy among MSCRAMMs. Judged from the increased binding profile of the RofA2 mutant, these factors were also under control of RofA. The deduced simultaneous positive and negative effects on the expression of various genes resembles the rofA6 but not the nra 49 regulatory profile.

The MSCRAMMs responsible for the observed binding profile remain obscure. The M2 strain was shown to possess a sof and a $f b p 54$ gene, both of which have been associated with fibronectin binding (Rakonjac et al., 1995; Kreikemeyer et al., 1995, 1999; Courtney et al., 1994, 1999; Katerov et al., 2000). However, transcription of both genes was unaltered in the RofA2 mutant. So far Cpa is the only collagen-binding MSCRAMM in GAS that has been characterized at the molecular level. Since M2 lacks Cpa it is likely that the M2 strain studied here harbours a novel collagen-binding mechanism.

Fibronectin binding due to protein $\mathrm{F}$ has been unequivocally associated with host cell attachment and internalization. Similarly, derepression or repression of fibronectin binding by protein $\mathrm{F}$ in Nra49 or RofA6 mutants led to increased or decreased cell attachment and internalization, respectively. However, the RofA2 mutant behaved unexpectedly. Despite its decreased fibronectin-binding activity it showed increased cell attachment and unchanged internalization. GAS host cell attachment and internalization independent of the prtF genes has been demonstrated before (Jaffe et al., 1996; Molinari et al., 2000, 2001). The present study indicates that such $p r t F$-independent mechanisms do not rely on a structural coupling of both processes, as it was demonstrated for the protein $\mathrm{F}$ function (Talay et al., 2001). The factors responsible for the increased affinity of the RofA2 mutant for HEp-2 cells remain uncharacterized. However, the decreased expression of genes involved in capsule synthesis in the mutant could contribute to the observed effects. Inactivation of the $h$ as operon in the M2 strain increased its adherence to keratinocytes by a factor of two (Darmstadt et al., 2000).

In summary, in three different GAS serotype strains examined for their RALP regulation pattern, differences were found in the expression of all but one virulence gene. Only $\operatorname{sag} A$, putatively encoding streptolysin $S$, was 
consistently derepressed in the RofA2, RofA6 and Nra49 mutants. Thus, not only the distribution of RALP genes and RALP-dependent MSCRAMM genes, but also the expression patterns of many virulence genes contained in RALP regulons exhibit a strain-specific variability. This variability clearly exceeds that of the regulons of the global Mga and CsrR regulators.

A potential association of this variability with the pathogenic potential of a given GAS serotype cannot be excluded in spite of the inconclusive results of the present investigation. However, a definitive answer necessitates the collection and molecular characterization of a very large number of GAS strains together with the information about their clinical background.

The analysis of rofA2-dependent gene expression resulted in an interesting side aspect. So far, rpsL-like ribosomal protein genes were assumed to be constitutively expressed. Now we could demonstrate that in GAS, rpsL transcription is under control of RofA2. Concerning the mutant's resistance to aminoglycosides, the decreased RpsL expression had the same effect as mutations in rpsL (Brakier-Gingras \& Phoenix, 1984) potentially by decreasing the number of targets for the antibiotics. However, changes in surface characteristics leading to decreased uptake of antibiotics may also account for the increased MIC.

This is the first demonstration of altered antibiotic resistance levels due to the activity of a virulence regulator in streptococci.

\section{ACKNOWLEDGEMENTS}

We thank A. Flosdorff and Y. Humboldt for expert technical assistance. S.B. and A.P. were supported by grant A09.22 of the IZKF, Ulm, Germany. B.K., A.B.-K. and A.P. were supported by DFG grant Po391/6-2, and A.P. was also supported by DFG grant Po391/3-4. We would like to thank Professor Tim Foster (Trinity College, Dublin, Ireland) for helpful advice on the manuscript.

\section{REFERENCES}

Ausubel, F. M., Brent, R., Kingston, R. E., Moore, D. D., Seidman, J. G., Smith, J. A. \& Struhl, K. (editors). (1990). Current Protocols in Molecular Biology. New York: Wiley.

Beckert, S., Kreikemeyer, B. \& Podbielski, A. (2001). Group A streptococcal rofA gene is involved in control of several virulence genes and eukaryotic cell attachment and internalization. Infect Immun 69, 534-537.

Bernish, B. \& van de Rijn, I. (1999). Characterization of a twocomponent system in Streptococcus pyogenes which is involved in regulation of hyaluronic acid production. J Biol Chem 274, 4786-4793.

Björkman, J., Samuelsson, P., Andersson, D. I. \& Hughes, D. (1999). Novel ribosomal mutations affecting translational accuracy, antibiotic resistance and virulence of Salmonella typhimurium. Mol Microbiol 31, 53-58.

Brakier-Gingras, L. \& Phoenix, P. (1984). The control of accuracy during protein synthesis in Escherichia coli and perturbations of this control by streptomycin, neomycin, or ribosomal mutations. Can J Biochem Cell Biol 62, 231-244.
Brandt, C. M., Spellerberg, B., Honscha, M., Truong, N. D., Hoevener, B. \& Lütticken, R. (2001). Typing of Streptococcus pyogenes strains isolated from throat infections in the region of Aachen, Germany. Infection 29, 163-166.

Caparon, M. G. \& Scott, J. R. (1991). Genetic manipulation of pathogenic streptococci. Methods Enzymol 204, 556-586.

Chaussee, M. S., Watson, R. O., Smoot, J. C. \& Musser, J. M. (2001). Identification of Rgg-regulated exoproteins of Streptococcus pyogenes. Infect Immun 69, 822-831.

Chen, C., Bormann, N. \& Cleary, P. P. (1993). VirR and Mry are homologous trans-acting regulators of $M$ protein and C5a peptidase expression in group A streptococci. Mol Gen Genet 241, 685-693.

Courtney, H. S., Li, Y., Dale, J. B. \& Hasty, D. L. (1994). Cloning, sequencing, and expression of a fibronectin/fibrinogen-binding protein from group A streptococci. Infect Immun 62, 3937-3946.

Courtney, H. S., Hasty, D. L., Li, Y., Chiang, H. C., Thacker, J. L. \& Dale, J. B. (1999). Serum opacity factor is a major fibronectinbinding protein and a virulence determinant of $M$ type 2 Streptococcus pyogenes. Mol Microbiol 32, 89-98.

Darmstadt, G. L., Mentele, L., Podbielski, A. \& Rubens, C. E. (2000). Role of group A streptococcal virulence factors in adherence to keratinocytes. Infect Immun 68, 1215-1221.

Federle, M. J., Mclver, K. S. \& Scott, J. R. (1999). A response regulator that represses transcription of several virulence operons in the group A Streptococcus. J Bacteriol 181, 3649-3657.

Ferretti, J. J., McShan, W. M., Ajdic, D. \& 20 other authors (2001). Complete genome sequence of an M1 strain of Streptococcus pyogenes. Proc Natl Acad Sci U S A 98, 4658-4663.

Fogg, G. C. \& Caparon, M. G. (1997). Constitutive expression of fibronectin binding in Streptococcus pyogenes as a result of anaerobic activation of rofA. J Bacteriol 179, 6172-6180.

Fogg, G. C., Gibson, C. M. \& Caparon, M. G. (1994). The identification of $r o f A$, a positive-acting regulatory component of $p r t F$ expression: use of an $\gamma \delta \mu$-based shuttle mutagenesis strategy in Streptococcus pyogenes. Mol Microbiol 11, 671-684.

Goodfellow, A. M., Hibble, M., Talay, S. R., Kreikemeyer, B., Currie, B. J., Sriprakash, K. S. \& Chhatwal, G. S. (2000). Distribution and antigenicity of fibronectin-binding proteins (SfbI and SfbII) of Streptococcus pyogenes clinical isolates from the northern territory, Australia. J Clin Microbiol 38, 389-392.

Granok, A. B, Parsonage, D., Ross, R. P. \& Caparon, M. G. (2000). The RofA binding site in Streptococcus pyogenes is utilized in multiple transcriptional pathways. J Bacteriol 182, 1529-1540.

Hanski, E. \& Caparon, M. G. (1992). Protein F, a fibronectinbinding protein, is an adhesin of the group A streptococcus Streptococcus pyogenes. Proc Natl Acad Sci U S A 89, 6172-6176.

Heath, A., DiRita, V. J., Barg, N. L. \& Engleberg, N. C. (1999). A two-component regulatory system, CsrR-CsrS, represses expression of three Streptococcus pyogenes virulence factors, hyaluronic acid capsule, streptolysin $\mathrm{S}$, and pyrogenic exotoxin $\mathrm{B}$. Infect Immun 67, 5298-5305.

Jaffe, J., Natanson-Yaron, S., Caparon, M. G. \& Hanski, E. (1996). Protein F2, a novel fibronectin-binding protein from Streptococcus pyogenes, possesses two binding domains. Mol Microbiol 21, 373-384.

Johnson, D. R. \& Kaplan, E. L. (1988). Microtechnique for serum opacity factor characterization of group A streptococci. J Med Microbiol 38, 311-315.

Katerov, V., Lindgren, P. E., Totolian, A. A. \& Schalen, C. (2000). Streptococcal opacity factor: a family of bifunctional proteins 
with lipoproteinase and fibronectin-binding activities. Curr Microbiol 40, 149-156.

Kaufhold, A., Podbielski, A., Johnson, D. R., Kaplan, E. L. \& Lütticken, R. (1992). M protein gene typing of Streptococcus pyogenes by nonradioactively labeled oligonucleotide probes. J Clin Microbiol 30, 2391-2397.

Kreikemeyer, B., Talay, S. R. \& Chhatwal, G. S. (1995). Characterization of a novel fibronectin-binding surface protein in group A streptococci. Mol Microbiol 17, 137-145.

Kreikemeyer, B., Martin, D. R. \& Chhatwal, G. S. (1999). SfbII protein, a fibronectin-binding surface protein of group A streptococci, is a serum opacity factor with high serotype-specific apolipoproteinase activity. FEMS Microbiol Lett 178, 305-311.

Kreikemeyer, B., Boyle, M. D. P., Buttaro, B. A., Heinemann, M. \& Podbielski, A. (2001). Group A streptococcal growth phaseassociated virulence factor regulation by a novel operon ( $f a s)$ with homologies to two-component-type regulators requires a small RNA molecule. Mol Microbiol 39, 392-406.

Levin, J. C. \& Wessels, M. R. (1998). Identification of $c s r R / c s r S$, a genetic locus that regulates hyaluronic acid capsule synthesis in group A Streptococcus. Mol Microbiol 30, 209-219.

Lukomski, S., Nakashima, K., Abdi, I., Cipriano, V. J., Ireland, R. M., Reid, S. D., Adams, G. G. \& Musser, J. M. (2000a). Identification and characterization of the $s c l$ gene encoding a group A Streptococcus extracellular protein virulence factor with similarity to human collagen. Infect Immun 68, 6542-6553.

Lukomski, S., Hoe, N. P., Abdi, I., Rurangirwa, J., Kordari, P., Liu, M., Dou, S. J., Adams, G. G. \& Musser, J. M. (2000b). Nonpolar inactivation of the hypervariable streptococcal inhibitor of complement gene (sic) in serotype M1 Streptococcus pyogenes significantly decreases mouse mucosal colonization. Infect Immun 68, 535-542.

Lukomski, S., Nakashima, K., Abdi, I., Cipriano, V. J., Shelvin, B. J., Graviss, E. A. \& Musser, J. M. (2001). Identification and characterization of a second extracellular collagen-like protein made by group A Streptococcus: control of production at the level of translation. Infect Immun 69, 1729-1738.

Lyon, W. R., Gibson, C. M. \& Caparon, M. G. (1998). A role for trigger factor and an Rgg-like regulator in the transcription, secretion and processing of the cysteine proteinase of Streptococcus pyogenes. EMBO J 17, 6263-6275.

Martin, N. J., Kaplan, E. L., Gerber, M. A., Menegus, M. A., Randolph, M., Bell, K. \& Cleary, P.P. (1990). Comparison of epidemic and endemic group $\mathrm{G}$ streptococci by restriction enzyme analysis. J Clin Microbiol 28, 1881-1886.

Mclver, K. S., Thurman, A. S. \& Scott, J. R. (1999). Regulation of $m g a$ transcription in the group A streptococcus: specific binding of Mga within its own promoter and evidence for a negative regulator. J Bacteriol 181, 5373-5383.

McLandsborough, L. A. \& Cleary, P. P. (1995). Insertional inactivation of the virR in Streptococcus pyogenes M49 demonstrates that VirR functions as a positive regulator of ScpA, FcrA, OF and M protein. FEMS Microbiol Lett 128, 45-52.

Molinari, G. \& Chhatwal, G. S. (1999). Streptococcal invasion. Curr Opin Microbiol 2, 56-61.

Molinari, G., Talay, S. R., Valentin-Weigand, P., Rohde, M. \& Chhatwal, G. S. (1997). The fibronectin-binding protein of Streptococcus pyogenes, SfbI, is involved in the internalization of group A streptococci by epithelial cells. Infect Immun $\mathbf{6 5}$, $1357-1363$.
Molinari, G., Rohde, M., Guzman, C. A. \& Chhatwal, G. S. (2000). Two distinct pathways for the invasion of Streptococcus pyogenes in non-phagocytic cells. Cell Microbiol 2, 145-154.

Molinari, G., Rohde, M., Talay, S. R., Chhatwal, G. S., Beckert, S. \& Podbielski, A. (2001). The role played by the group A streptococcal negative regulator $\mathrm{Nra}$ on bacterial interactions with epithelial cells. Mol Microbiol 40, 99-114.

Natanson, S., Sela, S., Moses, A. E., Musser, J. M., Caparon, M. G. \& Hanski, E. (1995). Distribution of fibronectin-binding proteins among group A streptococci of different $\mathrm{M}$ types. J Infect Dis 171, 871-878.

Okada, N., Tatsuno, I., Hanski, E., Caparon, M. G. \& Sasakawa, C. (1998). Streptococcus pyogenes protein $\mathrm{F}$ promotes invasion of HeLa cells. Microbiology 144, 3079-3086.

Ozeri, V., Rosenshine, I., Mosher, D. F., Fässler, R. \& Hanski, E. (1998). Roles of integrins and fibronectin in the entry of Streptococcus pyogenes into cells via protein F1. Mol Microbiol 30, 625-637.

Ozeri, V., Rosenshine, I., Ben-Ze'ev, A., Bokoch, G. M., Jou, T.-S. \& Hanski, E. (2001). De novo formation of focal complex-like structures in host cells by invading streptococci. Mol Microbiol 41, 561-573.

Patti, J. M., Allen, B. L., McGavin, M. J. \& Hook, M. (1994). MSCRAMM-mediated adherence of microorganisms to host tissues. Annu Rev Microbiol 48, 585-617.

Perez-Casal, J., Caparon, M. G. \& Scott, J. R. (1991). Mry, a transacting positive regulator of the $\mathrm{M}$ protein gene of Streptococcus pyogenes with similarity to the receptor proteins of twocomponent regulatory systems. J Bacteriol 173, 2617-2624.

Person, W. R., Wood, T., Zhang, Z. \& Miller, W. (1997). Comparison of DNA sequences with protein sequences. Genomics $\mathbf{4 6}$, 24-36.

Podbielski, A., Melzer, B. \& Lütticken, R. (1991). Application of polymerase chain reaction to study the $M$ protein(-like) gene family in beta-hemolytic streptococci. Med Microbiol Immunol 180, 213-227.

Podbielski, A., Kaufhold, A. \& Cleary, P. P. (1993). PCR-mediated amplification of Group A streptococcal genes encoding immunoglobulin-binding proteins. Immunomethods 2, 55-64.

Podbielski, A., Flosdorff, A. \& Weber-Heynemann, J. (1995). The group A streptococcal virR49 gene controls expression of four structural vir regulon genes. Infect Immun 63, 9-20.

Podbielski, A., Woischnik, M., Leonard, B. A. \& Schmidt, K. H. (1999). Characterization of $n r a$, a global negative regulator gene in group A streptococci. Mol Microbiol 31, 1051-1064.

Rakonjac, J. V., Robbins, J. C. \& Fischetti, V. A. (1995). DNA sequence of the serum opacity factor of group A streptococci: identification of a fibronectin-binding repeat domain. Infect Immun 63, 622-631.

Rasmussen, M. \& Björck, L. (2001). Unique regulation of SclB - a novel collagen-like surface protein of Streptococcus pyogenes. Mol Microbiol 40, 1427-1438.

Rasmussen, M., Eden, A. \& Björck, L. (2000). SclA, a novel collagen-like surface protein of Streptococcus pyogenes. Infect Immun 68, 6370-6377.

Shaw, J. H. \& Clewell, D. B. (1985). Complete nucleotide sequence of macrolide-lincosamide-streptogramin B-resistance transposon Tn917 in Streptococcus faecalis. J Bacteriol 164, 782-796.

Talay, S. R., Ehrenfeld, E., Chhatwal, G. S. \& Timmis, K. N. (1991). Expression of the fibronectin-binding components of Strepto- 
coccus pyogenes in Escherichia coli demonstrates that they are proteins. Mol Microbiol 5, 1727-1734.

Talay, S. R., Valentin-Weigand, P., Jerlström, P. G., Timmis, K. N. \& Chhatwal, G. S. (1992). Fibronectin-binding protein of Streptococcus pyogenes: sequence of the binding domain involved in adherence of streptococci to epithelial cells. Infect Immun 60, 3837-3844.

Talay, S. R., Valentin-Weigand, P., Timmis, K. N. \& Chhatwal, G. S. (1994). Domain structure and conserved epitopes of Sfb protein, the fibronectin-binding adhesin of Streptocccus pyogenes. Mol Microbiol 13, 531-539.

Talay, S. R., Zock, A., Rohde, M., Molinari, G., Oggioni, M., Pozzi, G., Guzman, C. A. \& Chhatwal, G. S. (2001). Co-operative binding of human fibronectin to Sfbl protein triggers streptococcal invasion into respiratory epithelial cells. Cell Microbiol 6, 521-535.

Toivonen, J. M., Boocock, M. R. \& Jacobs, H. T. (1999). Modelling in Escherichia coli of mutations in mitoribosomal protein S12: novel mutant phenotypes of rpsL. Mol Microbiol 31, 1735-1746. VanHeyningen, T., Fogg, G., Yates, D., Hanski, E. \& Caparon, M. (1993). Adherence and fibronectin binding are environmentally regulated in the group A streptococci. Mol Microbiol 9, 1213-1222.

Received 13 September 2001; revised 3 January 2002; accepted 14 January 2002. 